\title{
A estrutura do 16PF-5, versão espanhola: uma análise fatorial dos itens ${ }^{1}$
}

\author{
Valdiney V. Gouveia \\ Universidade Federal da Paraíba \\ José María Prieto \\ Universidade Complutense de Madri
}

\begin{abstract}
Resumo
O sistema de Cattell dos dezesseis fatores de primeira ordem no âmbito da personalidade normal não tem mudado desde seus estudos de elaboração há aproximadamente 50 anos. Este artigo examina a evidência que apoia a estrutura da versão espanhola do $16 \mathrm{PF}-5$, o qual foi aplicado a 3.446 pessoas e o conjunto de itens foi fatorizado diretamente. Foi realizada uma análise dos fatores principais, com rotação oblíqua direct quartimin e as matrizes fatoriais padrão e estrutura foram examinadas. As conclusões foram de que, embora a estrutura da personalidade seja mais clara quando as parcelas dos itens são consideradas (Cattell \& Cattell, 1995; Prieto, Gouveia, \& Fernández, 1996), os dezesseis fatores foram parcialmente confirmados analisandose unicamente os itens. Neste sentido, é apropriado pensar na versão espanhola do 16 PF-5 como uma medida adequada dos fatores da personalidade normal, como tem sido definida por R. B. Cattell.
\end{abstract}

Palavras-chave: 16PF; questionário; personalidade; validade de construto

\begin{abstract}
The structure of the 16PF-5, Spanish version: a factor analysis of items. The Cattell's system of sixteen primary factors in the normal personality sphere has not changed since its foundation studies approximately 50 years ago. This paper examines the evidence that supports the first order structure of the Spanish version of the 16 PF-5. This questionnaire was administered to 3,446 people, and the pool of items was factored. Principal factor solution and oblique rotation were performed. The sorted factor pattern and structure matrixes are available. The conclusion is that, although the structure of personality is more evident when the item parcels are considered (Cattell \& Cattell, 1995; Prieto, Gouveia, \& Fernández, 1996), all sixteen factors have been moderately confirmed analyzing only the items. So, it is appropriate to think of the Spanish version of 16 PF- 5 as an appropriate measurement of the normal personality factors, as it has been defined by R.B. Cattell.
\end{abstract}

Key words: 16PF; questionnaire; personality; construct validity

$\mathrm{O}$ questionário $16 \mathrm{PF}$ foi elaborado por Raymond $\mathrm{B}$. Cattell recorrendo a análises fatoriais sucessivas, refinando as dimensões principais identificadas inicialmente e depurando o conjunto de itens mantidos. A quinta edição, tanto em inglês como em espanhol, parece compreender um instrumento polido pronto para ser usado em pesquisa psicológica e na prática, em que o enfoque de análise são as características da personalidade normal. A primeira versão original deste questionário foi escrita e validada através de sucessivas análises fatoriais realizadas nos anos 1940; 30 anos depois, este foi considerado um instrumento útil entre os pesquisadores da personalidade, sendo também utilizado nas prá- ticas dos psicólogos clínicos, educacionais e organizacionais (Fabricatore, Azen, Schoentgen, \& Snibbe, 1978; Fox, Haboucha, \& Yossi, 1981; Lord, 1996; Seisdedos, 1996). É ampla a lista de versões disponíveis em diferentes idiomas e para períodos específicos do desenvolvimento humano.

Atualmente este questionário está presente em quase todas as culturas em que os psicólogos contribuem para a sociedade ajudando a melhorar a qualidade de vida das pessoas. A primeira versão espanhola do $16 \mathrm{PF}$ foi delineada, analisada, revisada e publicada por TEA Edições, durante os anos 70 (Cattell, 1975; Seisdedos, 1978). Na Tabela 1 é apresentado um resumo dos fatores de primeira ordem desse instrumento. 
Tabela 1

Descrição da Tipologia de Personalidade (16PF) de R. B. Cattell

\begin{tabular}{ll}
\hline \multicolumn{1}{c}{ Fator } & \multicolumn{1}{c}{ Atributos Principais } \\
\hline A (Expansivo) & Afetivo, condescendente, participante \\
B (Inteligente) & Pensamento abstrato, maior capacidade mental \\
C (Emocionalmente Estável) & Enfrenta a realidade, calmo, amadurecido \\
E (Afirmativo) & Independente, agressivo, obstinado \\
F (Despreocupado) & Impulsivamente animado, alegre, entusiasta \\
G (Consciencioso) & Perseverante, circunspeto, preso a normas \\
H (Desenvolto) & Desembaraçado, ousado, espontâneo \\
I (Brando) & Terno, dependente, superprotegido, sensível \\
L (Desconfiado) & Obstinado em sua opinião, difícil de enganar \\
M (Imaginoso) & Voltado para o interior, descuidado de assuntos práticos, boêmio \\
N (Requintado) & Esmerado, apurado, sagaz \\
O (Apreensivo) & Preocupado, deprimido, perturbado \\
Q1(Experimentador) & Renovador, criativo, liberal, analisador, mente aberta \\
Q2(Auto-suficiente) & Prefere próprias decisões, basta-se a si próprio \\
Q3(Controlado) & Socialmente correto, comandado por sua auto-imagem \\
Q4(Tenso) & Frustrado, impulsivo, irritado \\
\hline
\end{tabular}

Tyler (1996) descreve o 16PF não como um questionário elementar, mas como uma estrutura complexa; triando, identificando e mapeando as dimensões básicas da personalidade. A complexidade de cobrir a esfera total da personalidade, como refletida na proposta de Cattell (1946), é sem dúvida uma das questões que tem sido mais criticada e estado sob suspeita durante as cinco décadas de sua existência. Especificamente, o grau de adequação e representatividade dos dezesseis fatores identificados no âmbito da personalidade normal parte de dimensões psicológicas subjacentes duvidosas. Alguns autores afirmam que um número menor de fatores explicaria razoavelmente bem as covariâncias entre as escalas primárias do 16PF (Eysenck, 1972; Howarth \& Browne, 1971). Contrário a esta abordagem, Guilford (1975) descreve que

há indubitavelmente mais fatores de personalidade do que os clusters que Cattell supunha ter - uma situação impossível para as análises estatísticas [naqueles dias]. Com certeza ele esperava um número menor de dimensões, embora existam realmente muitas mais. (p. 810)

Cattell não descartava a sugestão de que entre 12 a 23 fatores primários poderiam ser necessários para descrever as personalidades de indivíduos anormais e normais, respectivamente. Até 1972, ele realizou um grande número de análises e gerou uma área ampla de questionários, enfatizando uma correspondência direta entre as escalas e as dimensões. Esta ênfase foi fundamentada em resultados concorrentes obtidos de 50 estudos realizados independentemente, que procuravam avaliar a convergência no número de fatores do 16PF: Questionário de Personalidade para Pré-Escolares $(P S P Q)$, Questionário de Personalidade para Ensino Fundamental (ESPQ), Questionário de Personalidade para Ensino Médio (HSPQ), Questionário de Personalidade para Crianças $(C P Q)$ e Questionário de Análise Clínica $(C A Q)$ (para uma revisão detalhada, ver Cattell, 1973). O número de fatores identificados através do Scree test coincidiu com o número de fatores fixados depois da solução de máxima verossimilhança (Cattell \& Krug, 1986; Prieto, 1980).

A respeito da estrutura do $16 \mathrm{PF}$ especificamente, Cattell e Krug (1986) comentam que

o número de fatores indicado é provavelmente maior se os 187 itens da Forma A do teste são diretamente fatorizados; algo menos se são dispostos em, digamos, 50 blocos razoavelmente homogêneos; e ainda menos se alguém considera o número mínimo de itens necessários (32) para definir os 16 fatores (por exemplo, dividindo ao meio cada uma das 16 escalas). Teoricamente, o número de fatores para os blocos seria o mesmo daqueles dos itens, mas devido aos erros de medida este número pode ser menor no caso dos primeiros. (p. 512)

Além da questão crítica do número adequado de fatores para descrever a estrutura da personalidade normal, muitos outros problemas foram levantados no campo da pesquisa em personalidade. Por exemplo, Guilford (1975), a respeito da elaboração do 16PF, levanta duas objeções: (1) os itens usados evidenciam cargas fatoriais baixas por fator, e (2) os itens heterogêneos saturam no mesmo fator, freqüentemente pon- 
tuando em direções opostas. Seguindo a perspectiva da psicometria convencional, unicamente itens homogêneos facilitam a segurança de uma solução fatorial com estrutura simples. De acordo com os ensinamentos básicos da análise fatorial, significaria escrever vários itens com certa similaridade, variando minimamente (por exemplo, "Você é cordial com colegas do seu grupo?", "Seus colegas e companheiros consideram que você é uma pessoa muito gentil no trabalho?"). Propositalmente, Cattell (1973) evitou introduzir itens semanticamente concretos, ao invés disso escolheu itens comportamentalmente concretos (por exemplo, "Você é amigável com um colega dissidente no escritório?", "Você tem êxito em estabelecer um clima de amizade com clientes habituais?"). Neste sentido, ele optou por itens complexos para descrever condutas da vida real procurando maneiras e padrões multifacetados.

As críticas de Guilford foram reforçadas por H. J. Eysenck (1972), bem como por vários outros membros daquele grupo de pesquisadores da personalidade; conjuntamente não concordavam com os fundamentos psicométricos do 16PF (ver, por exemplo, Bolton, 1977; Burdsal \& Bolton, 1979). Estes encontraram dificuldade para reproduzir independentemente a estrutura de personalidade colocada em lugar seguro por Cattell e seus colaboradores diretos no Institute for Personality and Ability Testing (IPAT). Analisaram o mesmo conjunto de itens do $16 \mathrm{PF}$, porém não seguiram os mesmos passos, procedimentos ou algoritmos na análise e rotação das matrizes fatoriais. Nos anos 70, a capacidade dos processadores e programas estatísticos informatizados deixava muito a desejar se comparados com os disponíveis nos últimos dez anos. Os padrões de antes refletiam máquinas e programas ad hoc, sendo operados em locais bastante específicos e restritos. O BDMP e o SPSS abriram as vias para padrões comerciais em análises multivariadas, eliminando os mitos em torno dos softwares que eram utilizados unicamente em cada centro de pesquisa separadamente.

Apesar das diversas formas do $16 \mathrm{PF}$ (A, B, C, D e E) e das versões desenvolvidas com a proposta de avaliar a personalidade em idade jovem (o $H S P Q$ e o $C P Q$ ), este instrumento continua leal à sua missão original de avaliar os fatores básicos subjacentes à personalidade normal, segundo o modelo descrito por R. B. Cattell. A publicação recente do 16PF-5 (ver Conn \& Rieke, 1994) é prova disto. É o resultado de um esforço de validação de suas diferentes formas, respeitando a estrutura de dezesseis dimensões de primeira ordem, e cinco de segunda ordem para a descrição da personalidade humana. Não obstante, pouca informação tem sido reunida sobre essa versão; além de Cattell e Cattell (1995), a principal fonte é um número especial da publicação European Review of Applied Psychology dedicado à mais nova edição do 16PF (ver Seisdedos, 1996).

Cattell e Cattell (1995) consideraram uma amostra diversificada de norte-americanos $(\mathrm{n}=3.498)$, distribuídos eqüitativamente quanto ao sexo. Cada escala teórica do $16 \mathrm{PF}$ foi composta pelos 14 melhores itens, como comprovado em estudos prévios. Estes foram reunidos em seis parcelas (blocos) de dois ou três itens em cada uma, segundo as correla- ções apresentadas entre eles. Posteriormente, uma análise fatorial foi realizada, utilizando a máxima correlação de cada parcela como a estimativa inicial da comunalidade. Os autores comentam que as parcelas, em lugar dos itens individuais, foram fatorizadas porque estas são muito mais precisas do que os itens. Embora unicamente dez dos fatores tenham apresentado eigenvalues iguais ou superiores a 1 , o número de fatores a ser extraído foi fixado em 20. Os resultados foram animadores:

Quatro dos 20 fatores foram indefinidos e assim não interpretáveis. No geral, o padrão fatorial revelou uma excelente estrutura simples. A maioria (94\%) das 95 parcelas apresentou cargas fatoriais de 0,30 ou superior em apenas um fator. As exceções foram a parcela $\mathrm{G} 2$, que teve uma segunda, porém menor, carga fatorial no fator O, e as parcelas A5, I1, I5 e O3, que apresentaram cargas fatoriais em um dos quatro fatores não definidos. (Cattell \& Cattell, 1995, p. 929)

Rolland e Mogenet (1996), com uma amostra de 1.000 participantes, 500 homens e 500 mulheres, com média de 32 anos de idade, estudaram a validade de construto (estrutura fatorial) e a consistência interna (Alfa de Cronbach) para a versão francesa do 16PF-5. Tratando com itens ao invés de parcelas (grupos de itens teoricamente homogêneos) e excluindo aquelas da escala $\mathrm{B}$, eles realizaram uma análise fatorial confirmatória do tipo multi-grupo, checando a similaridade entre a estrutura hipotética e a empiricamente extraída, que apoiou, naquele contexto, a descrição da personalidade como propõe Cattell. Das 15 escalas consideradas, exceto para a A que apresentou um Alfa de 0,57, todas as demais atenderam razoavelmente a demanda de valores aceitáveis (entre 0,62 e 0,81 ).

Prieto, Gouveia e Fernández (1996) também estudaram a estrutura primária do $16 \mathrm{PF}-5$ excluindo a escala $\mathrm{B}$, sendo que consideraram as parcelas de itens diretamente, ao invés dos itens. Sua amostra foi de mais de 3.000 participantes espanhóis, a maioria do sexo masculino. Através de uma análise fatorial PF (Principal Factor) com rotação direct quartimin, considerando o critério de carga fatorial igual ou superior a \pm 0,25 , eles observaram que as parcelas foram definidas claramente em 13 das 15 escalas; unicamente em duas delas $(\mathrm{C} \mathrm{e} \mathrm{G})$ uma das quatro parcelas não foi confirmada. Especificamente, $\mathrm{C} 1$ figurou na escala $\mathrm{O}$ com carga fatorial de $-0,45$, e G4 na escala Q3 com carga de 0,37. A conclusão dos autores foi de que na Espanha se confirmaram os fatores de primeira ordem que o $16 \mathrm{PF}-5$ procura medir.

Com sua quinta edição, o $16 \mathrm{PF}$ parece ter chegado ao ponto de maturidade proposto por Cattell. Provavelmente as próximas modificações, se ocorrerem, serão mais uma questão de adaptação cultural do que de estruturação teórica. Parece ser o momento para eliminar qualquer dúvida em torno do $16 \mathrm{PF}$ e assegurar sua efetividade psicométrica. $\mathrm{O}$ presente estudo tem como objetivo comprovar se é possível identificar a estrutura de personalidade proposta no $16 \mathrm{PF}-5$ analisando diretamente os itens como dados de entrada. Trata-se, portanto, de seguir os mesmos passos do seu autor, adotando procedimentos estatísticos equivalentes para verificar em 
que medida a estrutura resultante pode ser interpretada (ver Cattell, 1946, 1973). Segundo as observações de Cattell e Krug (1986, p. 512), resultados menos claros deveriam ser esperados do que aqueles encontrados por Rolland e Mogenet (1996), e Prieto, Gouveia e Fernández (1996).

\section{Método}

\section{Amostra}

A amostra deste estudo foi composta de 3.446 participantes, provenientes dos seguintes contextos: seleção de pessoal, programa de orientação vocacional entre adultos no serviço militar (é compulsório na Espanha), escolas técnicas ou universidades. A média de idade destes foi de 19 anos, sendo a maioria do sexo masculino $(81,3 \%)$.

\section{Instrumento}

O instrumento utilizado foi uma versão experimental da forma espanhola do 16PF 5ª Edição (Russell \& Karol, 1995), constando de 200 itens. Destes, 185 correspondiam à versão original norte-americana (Estados Unidos) e os outros 15 itens foram obtidos da versão espanhola do 16PF Forma A. Neste caso, considerou-se o melhor item de cada um dos 15 fatores (a escala B foi excluída), considerando o critério de saturação observado em estudos prévios (Prieto, 1980; Seisdedos, 1978). Este conjunto de itens foi distribuído aleatoriamente no questionário aplicado.

\section{Procedimento}

Embora o questionário tenha sido respondido em diferentes contextos, tomou-se o cuidado de seguir instruções padronizadas para a coleta dos dados, como podem ser encontradas no seu manual técnico (ver Conn \& Rieke, 1994). Os participantes responderam este instrumento individualmente, mas predominantemente em situação coletiva, entregando-o imediatamente ao seu aplicador. Este co- nhecia as normas de aplicação e tinha recebido um treino prévio para tanto.

\section{Tabulação e análise dos dados}

As folhas de resposta dos participantes foram digitalizadas e suas pontuações brutas para cada item armazenadas. Posteriormente, estas foram transformadas, adotando o padrão de correção elaborado para a versão espanhola do 16PF-5. O banco de dados resultante foi depurado, eliminando-se os casos omissos e os valores extremos.

Para a análise dos dados, foi utilizada a versão de 1990 do pacote estatístico BMDP. Além da distribuição de frequiência e outras estatísticas descritivas empregadas, foi realizada uma análise fatorial para comprovar a estrutura do 16PF. Optou-se por uma análise de Fatores Principais e pelo procedimento de rotação direct quartimin (Jenrich \& Sampson, 1966), que é teoricamente adequado devido à natureza oblíqua dos fatores neste instrumento (Tyler, 1996). Inicialmente foi comprovado que a maioria das comunalidades das variáveis foi inferior a 0,50 . Neste caso, o quadrado da correlação múltipla de cada uma com as demais foi escolhido como o elemento mais apropriado, evitando-se superestimar os valores da diagonal da matriz de correlações (Gorsuch, 1983).

\section{Resultados}

Primeiramente, para conhecer o número de fatores a serem extraídos da matriz de pontuações corrigidas, foram examinados os eigenvalues. O Scree test pareceu ser o procedimento mais apropriado; estima-se que em amostras estatisticamente grandes, como a considerada no presente estudo, sua configuração não é afetada (Cattell \& Krug, 1986). Sendo menos ortodoxo e com o fim de maximizar o número de fatores resultantes, decidiu-se não seguir a rule of thumb que estabelece um eigenvalue de 1 para a identificação da estrutura fatorial; no presente caso, assumiu-se um valor de 0,50. Os resultados são descritos a seguir na Figura 1.

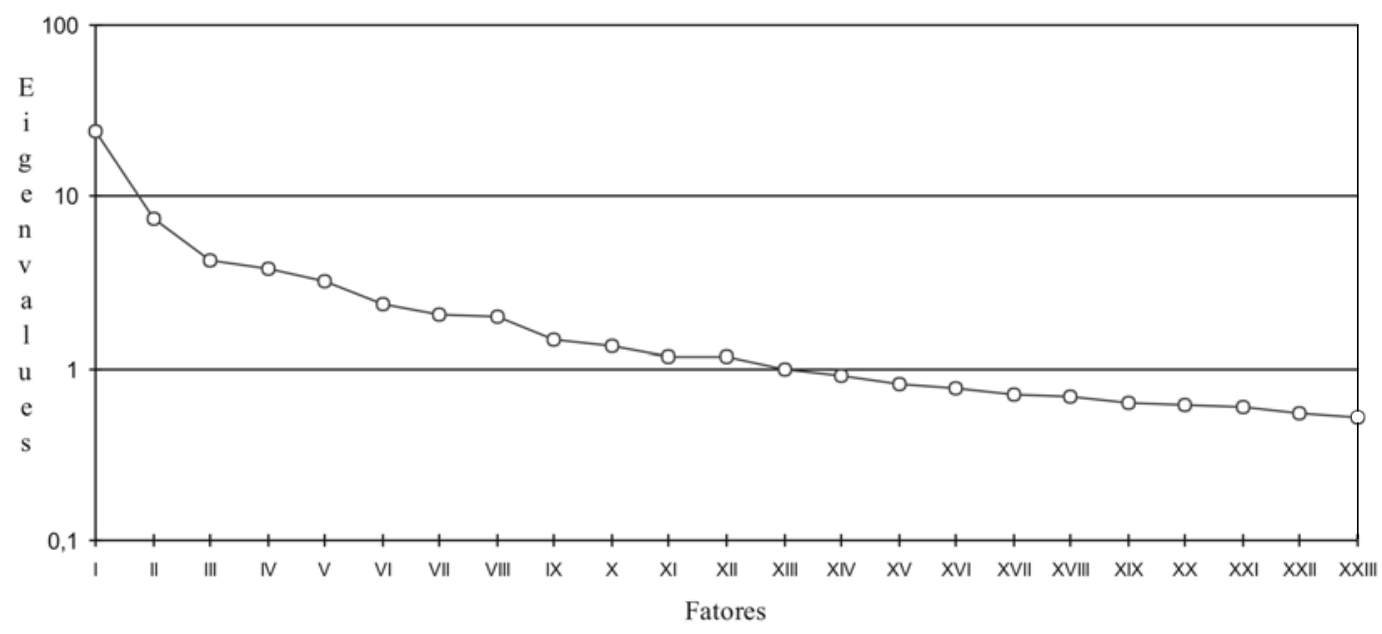

Figura 1. Representação gráfica dos eigenvalues para os primeiros 23 fatores não rotados. 
Como pode ser observado nessa figura, até um total de 23 fatores puderam ser extraídos seguindo o critério antes descrito. Deste grupo, foram identificados 12 ou 13 fatores com eigenvalues próximos a 1. O fator 16 apresentou um eigenvalue de 0,78 , parecendo razoável tê-lo em consideração como um critério de extração dos fatores nas análises posteriores.

Embora os participantes tenham respondido aos 200 itens da versão espanhola do 16PF-5, doze destes itens ficaram fora da análise fatorial, correspondendo aos da escala de Impression Management (IM). Com o critério de extração de 16 fatores com eigenvalue igual ou superior a 0,78 , os resultados obtidos são apresentados na Tabela 2.

Na Tabela 2 figuram unicamente os dados em relação ao comportamento de cada item que melhor descreve o fator e a abreviação da escala a qual teoricamente pertence. A matriz de referência neste estudo é a padrão; não obstante, as saturações da matriz estrutura são também disponibilizadas por-

Tabela 2

Estrutura Fatorial do $16 P F-5$, Versão Espanhola $(n=3.446)$

\begin{tabular}{|c|c|c|c|c|c|c|c|c|}
\hline Item & \multicolumn{2}{|c|}{$\mathbf{H}$} & \multicolumn{2}{|c|}{ Q2 } & \multicolumn{2}{|c|}{ O } & \multicolumn{2}{|c|}{ L } \\
\hline $\begin{array}{c}1 \\
2 \\
3 \\
4 \\
5 \\
6 \\
7 \\
8 \\
9 \\
10 \\
11 \\
\end{array}$ & $\begin{array}{l}72 \\
72 \\
66 \\
63 \\
61 \\
60 \\
57 \\
56 \\
52 \\
43 \\
43 \\
\end{array}$ & $\begin{array}{l}79 \\
77 \\
72 \\
70 \\
70 \\
67 \\
66 \\
\mathbf{6 6} \\
59 \\
53 \\
52\end{array}$ & $\begin{array}{l}84 \\
82 \\
55 \\
47 \\
46 \\
40 \\
33 \\
28 \\
27 \\
26 \\
-\end{array}$ & $\begin{array}{l}82 \\
80 \\
63 \\
55 \\
48 \\
49 \\
48 \\
39 \\
39 \\
37 \\
31\end{array}$ & $\begin{array}{l}59 \\
58 \\
57 \\
43 \\
39 \\
39 \\
38 \\
32 \\
31 \\
27 \\
-\end{array}$ & $\begin{array}{l}67 \\
68 \\
60 \\
60 \\
54 \\
49 \\
48 \\
51 \\
51 \\
24 \\
33 \\
\end{array}$ & $\begin{array}{l}61 \\
59 \\
52 \\
49 \\
46 \\
44 \\
44 \\
39 \\
= \\
\end{array}$ & $\begin{array}{r}\mathbf{6 5} \\
\mathbf{6 4} \\
\mathbf{5 7} \\
\mathbf{5 5} \\
\mathbf{5 4} \\
\mathbf{5 1} \\
\mathbf{5 2} \\
\mathbf{4 5} \\
\mathbf{3 3} \\
\mathbf{3 0} \\
\mathbf{- 0 1} \\
\end{array}$ \\
\hline Item & \multicolumn{2}{|c|}{ Q3 } & \multicolumn{2}{|c|}{ I } & \multicolumn{2}{|c|}{ C } & \multicolumn{2}{|c|}{$\mathbf{N}$} \\
\hline $\begin{array}{c}1 \\
2 \\
3 \\
4 \\
5 \\
6 \\
7 \\
8 \\
9 \\
10 \\
11 \\
12 \\
\end{array}$ & $\begin{array}{l}58 \\
56 \\
51 \\
45 \\
44 \\
43 \\
42 \\
38 \\
32 \\
25 \\
--\end{array}$ & $\begin{array}{l}\mathbf{5 7} \\
57 \\
53 \\
\mathbf{4 5} \\
52 \\
47 \\
50 \\
\mathbf{4 5} \\
\mathbf{4 6} \\
\mathbf{3 4} \\
\mathbf{2 6}\end{array}$ & $\begin{array}{l}61 \\
56 \\
56 \\
50 \\
47 \\
47 \\
41 \\
40 \\
36 \\
31 \\
28 \\
--\end{array}$ & $\begin{array}{l}\mathbf{6 6} \\
\mathbf{6 0} \\
\mathbf{5 8} \\
\mathbf{5 1} \\
\mathbf{5 3} \\
\mathbf{5 0} \\
\mathbf{4 6} \\
\mathbf{4 5} \\
\mathbf{3 8} \\
\mathbf{3 3} \\
\mathbf{2 8} \\
\mathbf{2 4} \\
\end{array}$ & $\begin{array}{l}51 \\
48 \\
42 \\
39 \\
37 \\
36 \\
35 \\
-- \\
-- \\
--\end{array}$ & $\begin{array}{l}66 \\
59 \\
58 \\
49 \\
48 \\
52 \\
47 \\
40 \\
37 \\
35 \\
35\end{array}$ & $\begin{array}{l}66 \\
61 \\
61 \\
54 \\
54 \\
40 \\
28 \\
= \\
= \\
=-\end{array}$ & $\begin{array}{r}\mathbf{6 8} \\
\mathbf{6 5} \\
\mathbf{6 1} \\
\mathbf{5 8} \\
\mathbf{5 7} \\
\mathbf{4 7} \\
\mathbf{3 8} \\
\mathbf{3 1} \\
\mathbf{2 8} \\
\mathbf{2 0} \\
\mathbf{- 0 5}\end{array}$ \\
\hline Item & \multicolumn{2}{|c|}{$\mathbf{F}$} & \multicolumn{2}{|c|}{ G } & \multicolumn{2}{|c|}{ B } & \multicolumn{2}{|c|}{$\mathbf{A}$} \\
\hline $\begin{array}{l}1 \\
2 \\
3 \\
4 \\
5 \\
6 \\
7 \\
8 \\
9 \\
10 \\
11 \\
12 \\
13 \\
14 \\
15 \\
\end{array}$ & $\begin{array}{l}63 \\
59 \\
52 \\
51 \\
32 \\
29 \\
- \\
-- \\
-- \\
--\end{array}$ & $\begin{array}{l}62 \\
65 \\
57 \\
53 \\
44 \\
37 \\
30 \\
29 \\
24 \\
21 \\
20\end{array}$ & $\begin{array}{l}44 \\
44 \\
43 \\
41 \\
40 \\
37 \\
30 \\
29 \\
27 \\
-- \\
--\end{array}$ & $\begin{array}{l}51 \\
46 \\
53 \\
52 \\
47 \\
42 \\
46 \\
40 \\
35 \\
36 \\
30 \\
20\end{array}$ & $\begin{array}{l}43 \\
40 \\
39 \\
38 \\
37 \\
30 \\
29 \\
28 \\
25 \\
-- \\
-- \\
-- \\
-- \\
-\end{array}$ & $\begin{array}{r}45 \\
41 \\
40 \\
40 \\
37 \\
31 \\
29 \\
29 \\
25 \\
21 \\
20 \\
19 \\
14 \\
-09 \\
04 \\
\end{array}$ & $\begin{array}{l}62 \\
59 \\
56 \\
51 \\
42 \\
= \\
= \\
= \\
= \\
=\end{array}$ & $\begin{array}{l}\mathbf{6 4} \\
\mathbf{6 1} \\
\mathbf{5 5} \\
\mathbf{5 0} \\
\mathbf{5 1} \\
37 \\
\mathbf{2 9} \\
\mathbf{1 9} \\
\mathbf{1 1} \\
\mathbf{0 7} \\
\mathbf{0 3} \\
\mathbf{0 2}\end{array}$ \\
\hline Item & \multicolumn{2}{|c|}{ Q1 } & \multicolumn{2}{|c|}{ Q4 } & \multicolumn{2}{|c|}{$\mathbf{E}$} & \multicolumn{2}{|c|}{ M } \\
\hline $\begin{array}{l}1 \\
2 \\
3 \\
4 \\
5 \\
6 \\
7 \\
8 \\
9 \\
10 \\
11 \\
12 \\
13 \\
14 \\
15 \\
\end{array}$ & $\begin{array}{l}50 \\
46 \\
32 \\
31 \\
29 \\
28 \\
26 \\
26 \\
-- \\
-- \\
-- \\
-- \\
--\end{array}$ & $\begin{array}{l}\mathbf{5 3} \\
49 \\
33 \\
38 \\
31 \\
36 \\
36 \\
27 \\
25 \\
22 \\
22 \\
11 \\
10 \\
07 \\
02\end{array}$ & $\begin{array}{l}48 \\
43 \\
34 \\
29 \\
28 \\
27 \\
- \\
- \\
-- \\
--\end{array}$ & $\begin{array}{l}52 \\
45 \\
40 \\
35 \\
33 \\
32 \\
31 \\
28 \\
28 \\
24 \\
18\end{array}$ & $\begin{array}{l}55 \\
55 \\
50 \\
26 \\
25 \\
- \\
- \\
- \\
- \\
--\end{array}$ & $\begin{array}{l}57 \\
54 \\
49 \\
29 \\
28 \\
28 \\
26 \\
26 \\
17 \\
15 \\
04\end{array}$ & $\begin{array}{l}= \\
= \\
= \\
= \\
= \\
=\end{array}$ & $\begin{array}{r}37 \\
36 \\
30 \\
30 \\
28 \\
24 \\
24 \\
21 \\
20 \\
17 \\
-12 \\
07\end{array}$ \\
\hline
\end{tabular}

Nota: Os coeficientes em negrito são cargas fatoriais da matriz structure; os pontos decimais foram omitidos. 
que compreendem coeficientes de correlação, podendo ser úteis para checar a adequação de cada item no grupo da escala, e posteriormente decidir sobre sua própria existência e correspondência com um determinado fator. Finalmente, é necessário dizer que os fatores são apresentados e descritos seguindo uma ordem decrescente de contribuição para a explicação da variância total das pontuações no instrumento, e que os itens com carga fatorial menor do que $\pm 0,25$, na matriz padrão, foram considerados irrelevantes na definição dos fatores.

Os 16 fatores resultantes são descritos a seguir. Em 13 deles, identificados com as etiquetas teóricas de $\mathbf{H}, \mathbf{Q} 2, \mathbf{O}, \mathbf{L}$, Q3, I, C, N, F, G, B, Q1 e Q4, foi comprovado que mais da metade dos seus respectivos itens apresentaram cargas fatoriais acima do pré-estabelecido. Para facilitar a interpretação dos fatores resultantes por parte do leitor, após a identificação da escala correspondente, são listados dois itens representativos:

Fator I - Este fator explicou $4,82 \%$ da variância. A menor saturação foi 0,43 , e a maior 0,72 ; nenhum dos seus onze itens saturou em outra escala além da $\mathbf{H}$ (desenvolto; "Não é problema para mim começar conversações com estranhos", "Considero-me uma pessoa socialmente comunicativa e muito atrevida"), que é clara por seu conteúdo.

Fator II - Uma variância total de 3,62\% foi explicada por este fator. Dos onze itens que descrevem a escala Q2 (auto-suficiente; "Geralmente gosto de fazer meus planos sozinho, sem interrupções e sugestões dos outros", "Consigo terminar as coisas melhor quando trabalho sozinho do que quando o faço em equipe"), dez apresentaram cargas fatoriais que oscilaram de 0,26 a 0,84. Não obstante, dois dos itens desta escala saturaram negativa e moderadamente no Fator IX. Especificamente, o item 4 com saturação de - 0,26, e o 9 com - 0,31. Fator III - 3,60\% da variância total foram explicados por este fator. Dez dos onze itens da escala $\mathbf{O}$ (apreensivo; "Comumente tendo a ser demasiado sensível e me preocupo muito em relação a algo que tenho feito", "Geralmente, depois de tomar uma decisão sobre algo, sigo pensando se será certa ou errada") saturaram com valores entre 0,27 e 0,59. Porém, os itens 9 e 10 que teoricamente pertencem à escala Q4 (tenso) também apresentaram cargas fatoriais positivas neste fator: 0,36 e 0,32 , respectivamente. $\mathrm{O}$ item 10 da escala $\mathbf{O}$ (apreensivo) apresentou uma carga fatorial de - 0,26 no Fator XVI.

Fator IV - A variância explicada por este fator foi de 3,58\%. A escala $\mathbf{L}$ (desconfiado; "Geralmente existe uma grande diferença entre o que as pessoas dizem e o que fazem", "Se a pessoa é franca e aberta os outros tentam se aproveitar dela") parece corresponder ao seu conteúdo, visto que oito dos seus onze itens apresentaram cargas fatoriais que variaram de 0,31 a 0,61 . Dois itens da escala $\mathbf{N}$ (requintado) foram também definidos neste fator: o 7, com saturação de 0,33 , e o 10 com 0,31; o item 7 também apresentou saturação acima da pré-estabelecida em sua escala correspondente, definida como Fator VIII. O três itens restantes da escala $\mathbf{L}(9,10$ e 11) não foram definidos em nenhum outro fator.

Fator $V-3,35 \%$ da variância total foram explicados por este fator. Dos onze itens da escala Q3 (controlado; "Não consigo estar à vontade em um ambiente desorganizado", "Sempre mantenho meus pertences em perfeitas condições"), dez saturaram neste fator com valores entre 0,25 e 0,58 . O item 11 desta escala não apareceu em nenhum outro fator, nem a saturação de algum outro item foi observada neste fator.

Fator VI - Este fator explicou 3,29\% da variância total. Dos seus doze itens, a escala I (brando; "Quando era uma criança empregava a maior parte do meu tempo em ler ou imaginar coisas ideais", "Emocionam-me as boas novelas ou obras de teatro/cinema") foi capaz de reunir onze com cargas fatoriais que variaram de 0,28 a 0,61. O item 12 desta escala não apresentou carga fatorial satisfatória em nenhum outro fator, e o 7 saturou com 0,41 no Fator VII.

Fator VII - 2,88\% da variância explicada foram produzidos por este fator. Um total de sete dos onze itens que compõem a escala C (emocionalmente estável; "Quando coisas sem importância saem mal uma atrás da outra, continuo de um modo normal", "Na minha vida pessoal, quase sempre alcanço as metas a que me proponho") saturaram neste fator com cargas que variaram de 0,35 a 0,51 . Três dos itens desta escala (3, 9 e 10) apresentaram cargas fatoriais negativas no Fator III, oscilando entre - 0,33 e - 0,40; dois dos seus itens ( 8 e 11) não foram definidos em nenhum outro fator.

Fator VIII - Este fator foi responsável pela explicação de $2,79 \%$ da variância total. Ele foi interpretado como sendo a escala N (requintado; "Resulta-me fácil falar sobre minha vida, inclusive sobre aspectos que outros considerariam muito pessoais", "Algumas pessoas crêem que é difícil chegar a me conhecer"), uma vez que sete dos onze itens desta escala apresentaram cargas fatoriais neste fator que variaram de 0,28 a 0,66. Como antes foi indicado, o item 7 também saturou no Fator III, e o 10 apresentou uma carga fatorial exclusiva neste fator. Três dos itens da escala $\mathbf{N}(8,9$ e 11) não figuraram em nenhum dos 16 fatores.

Fator IX - 2,35\% da variância total foram explicados por este fator. A escala $\mathbf{F}$ (despreocupado; "Creio mais em seguir quase sempre o dito 'divertir-se e ser feliz", "Geralmente gosto de estar no meio de muita animação e atividade"), composta por onze itens, foi representada por seis itens com cargas fatoriais acima de 0,25 no presente fator. Um dos itens desta escala, o de número 9 , saturou positivamente $(0,33)$ apenas no fator que será descrito a seguir. Os quatro itens restantes da escala $\mathbf{F}$ (7, 8, 10 e 11) não apresentaram cargas fatoriais aceitáveis em nenhum outro fator.

Fator $X$ - A explicação de 2,24\% da variância foi devida a este fator. Dos doze itens que definem a escala $\mathbf{G}$ (consciencioso; "Prefiro um lar onde se sigam as normas estritas de conduta", "Admiro mais uma pessoa com capacidade média, porém com uma moral estrita"), nove saturaram satisfatoriamente, com cargas que variaram de 0,27 a 0,44 . Os três itens restantes desta escala foram distribuídos entre os fatores IV (item 10, carga-0,27) e V (item 11, carga 0,53, e item 12, carga 0,29).

Fator XI - Este fator foi responsável pela explicação de 2,06\% da variância total. Os nove itens que o definiram, com cargas fatoriais de 0,25 a 0,43 , pertencem à escala $\mathbf{B}$ (inteligente; “'Minuto' está para a 'hora' como 'segundo' está para o 'mi- 
nuto’”, “"Melhor' está para 'péssimo' como 'pior' está para "ótimo"”); os seis itens que complementam esta escala não apresentaram cargas fatoriais em nenhum outro fator, segundo o valor pré-estabelecido como sendo satisfatório.

Fator XII - 2,02\% da variância total foram explicados por este fator. Dos doze itens que definem a escala A (expansivo; "Preferiria estar em uma oficina, organizando e atendendo a pessoas", "Desfruto ajudando as pessoas no que necessitem"), cinco saturaram neste fator com cargas que foram de 0,42 a 0,62. Dois itens desta escala, os de número 8 e 11, apresentaram saturações negativas no Fator VIII $(-0,30$ e $-0,33$, respectivamente), e outro item, o de número 6, no Fator II ($0,42)$; os quatro itens restantes não foram definidos satisfatoriamente em nenhum dos demais fatores.

Fator XIII - 1,99\% da variância total foram explicados por este fator. Parece apropriado defini-lo como a escala Q1 (experimentador; "Prefiro tentar novos modos de fazer as coisas que seguir métodos já conhecidos", "Estou mais interessado em buscar um significado pessoal na vida do que obter um trabalho com um bom salário”), uma vez que, dos 15 itens que a compõem, oito saturaram neste fator com cargas que variaram de 0,26 a 0,50 . $\mathrm{O}$ item número 12 desta escala apresentou saturação positiva no Fator XV $(0,34)$, e os seis itens restantes não alcançaram uma carga fatorial de $\pm 0,25$ em nenhum dos demais fatores.

Fator XIV - Este fator explicou 1,89\% da variância total. A escala Q4 (tenso; "Resulta-me difícil ser paciente quando as pessoas me criticam”, "Às vezes me frustro muito rápido com as pessoas") concentrou a maioria dos itens com saturações satisfatórias (seis de onze); a menor carga fatorial foi 0,27 , e a maior 0,48; um dos itens desta escala (o de número 6) saturou exclusiva e positivamente no Fator III $(0,38)$, e outro (o de número 11) saturou negativamente no VII $(-0,27)$. Três dos seus itens não apresentaram uma carga fatorial igual ou superior ao estabelecido em nenhum dos demais fatores.

Fator $X V-1,84 \%$ da variância total foram explicados por este fator. Parece razoável assumir que corresponde à escala $\mathbf{E}$ (afirmativo; "Nas situações nas que sou responsável me sinto bem dando instruções aos demais", "Os demais me consideram uma pessoa que sabe se impor"). De fato, dos onze itens desta escala, cinco se concentraram neste fator com cargas fatoriais que variaram de 0,25 a 0,55 . Dois itens desta escala (7 e 10) figuraram no Fator XVI, com cargas de 0,30 e 0,31 , respectivamente; e quatro dos seus itens não saturaram em nenhum outro fator.

Fator XVI - Este fator explicou 1,51\% da variância total. Não pareceu claro a que escala correspondia; seria esperado que correspondesse à escala M (imaginoso; "Meus amigos me consideram uma pessoa algo abstraída e pouco prática", "Geralmente meus pensamentos são demasiado complicados e profundos para serem compreendidos por muita gente"), mas os itens desta escala com cargas fatoriais próximas a $\pm 0,25$ foram distribuídos em seis fatores diferentes: item 1, carga 0,37, Fator XIII; item 6, carga 0,34, Fator VI; itens 7 e 12, cargas -0,26, Fator V; item 9, carga 0,28, Fator VI, e carga 0,28, Fator VII; e item 11, carga - 0,37, Fator IV. Quatro itens desta escala não apresentaram cargas fatoriais satisfatórias em nenhum outro fator.

Em resumo, se a abordagem de 50\% + 1 item é adotada para definir a confirmação empírica de uma escala teórica, das 16 escalas do 16PF-5, em 13 (H, Q2, O, L, Q3, I, C, N, F, G, B, Q1 e Q4) a tipologia de R.B. Cattell parece ser corroborada. Em duas escalas (A e E), embora este critério não seja atendido, observou-se que o número de itens presentes no fator que define, cinco em cada uma, somados aos que estão um pouco abaixo das cargas fatoriais pré-estabelecidas, correspondem à maioria dos itens analisados. Finalmente, ao menos na matriz padrão, a escala $\mathbf{M}$ não pôde ser identificada no conjunto de fatores extraídos.

\section{Discussão}

Contrariamente às reações negativas ao modelo de personalidade de R. B. Cattell (ver Adcock, Adcock, \& Walkey, 1974; Eysenck, 1972; Howarth, Browne, \& Marceu, 1972), em certa medida o presente estudo indica um suporte razoável à sua adequação. No conjunto total, parece plausível assumir a identificação da estrutura do 16 PF em sua versão mais recente; a maioria dos itens emergiu em pelo menos 13 dos seus fatores respectivos. Em todo caso, é importante que não se perca de vista que as análises efetuadas não tiveram um caráter confirmatório, no sentido que estabelecem os modelos de equações estruturais, e que alguns dos critérios convencionados na prática do psicometrista (por exemplo, eigenvalue maior ou igual a 1 , carga fatorial a partir de $|0,30|$ ) não foram atendidos. Esta situação provavelmente evidencia uma das limitações principais deste estudo e sugere novas pesquisas sobre a adequação desta medida e do modelo teórico que a fundamenta.

É possível que assumir um procedimento default para identificar uma estrutura fatorial revele muitas vezes a ausência de um modelo teórico axiomatizado, defendido em psicometria (Pasquali, 1999), e reduza toda prática ao estrito empirismo. Não obstante, seria imprudente deixar de reconhecer que, por serem estes critérios estatísticos externos e comumente aceitos, podem funcionar como crivos objetivos na tomada de decisão, inclusive permitindo comparar estruturas fatoriais observadas em diferentes culturas. Portanto, caberia uma discussão mais pormenorizada dos critérios (teóricos vs. estatísticos) para decidir sobre a validade de construto de uma medida, o que supera o escopo deste estudo.

Faz-se indispensável aqui compreender o conjunto total dos resultados observados, começando pela escala $\mathbf{M}$, que não se mostrou clara nos dados da matriz padrão. Esta pode ser pensada como correspondendo ao Fator XVI. Tome-se como referência a inspeção da matriz estrutura, em que, exceto para um dos 12 itens (o de número 11), todos os demais apresentaram cargas fatoriais positivas; nove dos quais com valores iguais ou superiores a 0,20 , sendo que cinco tiveram saturações entre 0,28 e 0,37 , todas sendo consideradas correlações significativas $(p<0,05)$. 
A respeito da dificuldade de comprovar a escala $\mathbf{M}$, foi realizado um estudo com 1.889 pessoas da Nova Zelândia, a maioria das quais homens com idades entre 20 e 50 anos, que responderam a Forma A do 16PF. Depois de realizar uma análise fatorial com rotação oblíqua, tratando diretamente com os itens, comprovou-se que esta escala não poderia ser definida como uma das melhores (ver Adcock \& Adcock, 1977). Prieto, Gouveia e Fernández (1996), embora tenham sido capazes de identificar claramente a escala $\mathbf{M}$ tomando como referência as parcelas dos itens, descrevem que entre todas as escalas avaliadas do 16PF-5, esta foi a que apresentou a porcentagem mais baixa de explicação da variância total.

É possível que o aspecto imaginoso da escala $\mathbf{M}$ (com itens como: "Sou o tipo de pessoa que imagino ou penso coisas sobre mim mesmo" ou "Às vezes estou tão envolvido nos meus pensamentos que, a não ser que saia deles, perco a noção do tempo e desordeno ou não encontro minhas coisas") defina uma variável complexa no sentido descrito por Guilford (1975). Claramente, com freqüência são obtidas cargas fatoriais baixas quando os itens desta escala são considerados. Em alguns casos, sua existência é mais evidente quando são levadas em conta as parcelas dos itens (Cattell \& Cattell, 1995; Prieto, Gouveia, \& Fernández, 1996), e é inquestionável quando seus itens individuais são fatorizados por grupo com independência das demais escalas, apresentando inclusive um dos melhores coeficientes de consistência interna entre todos os fatores do 16 PF-5 (Rolland \& Mogenet, 1996).

Com respeito às escalas $\mathbf{A}$ e $\mathbf{E}$, pareceram corresponder a dois dos 16 fatores extraídos, os números XII e XV, respectivamente. Não obstante, do número total de itens que teoricamente pertence a estas escalas, menos da metade apresentaram cargas fatoriais que atendem ao valor pré-estabelecido $( \pm$ 0,25). Considerando separadamente os dois grupos de itens que formam estas escalas, Rolland e Mogenet (1996) encontraram índices de consistência interna baixos: Alfa de Cronbach de 0,57 para a escala A, e de 0,65 para a E. Possivelmente isso reflita que nem todos os itens destas escalas são igualmente adequados para medir os construtos a que se propõem.

Considerando o grupo das escalas, foi comprovado que quatro delas (B, H, I e Q3) apresentaram cargas fatoriais apenas no fator que definem. Outras três $(\mathbf{Q 2}, \mathbf{C}$ e $\mathbf{A})$ tiveram itens que saturaram com cargas fatoriais negativas em outro fator e as demais escalas apresentaram ao menos um item com carga fatorial positiva em um fator diferente daquele a que teoricamente pertence. Este fato não contradiz a conclusão de que o 16PF-5 é uma medida apropriada das características da personalidade normal. O que sugere provavelmente, como pode ser deduzido de Tyler (1996), é a estrutura psicológica complexa que identifica e mapeia tal instrumento. Não é possível conceber a personalidade humana como fatores independentes, isto porque é muito difícil definir escalas com itens exclusivos; o surgimento dos Big Five como um modelo empírico para conhecer a personalidade é uma evidência deste aspecto (ver Cattell, 1996). Por certo, pesquisas futuras deveriam se centrar nesse modelo; poderia ser questionado em que medida estes cinco grandes fatores da personalidade emergem de medidas clássicas na literatura (16PF, MMPI, EPPS, etc.), ou mesmo se são interpretados mais adequadamente como os cinco fatores principais de primeira ordem ou aqueles de segunda ordem.

Se os resultados do presente estudo são comparados com os descritos por Prieto, Gouveia e Fernández (1996), pode ser comprovado que a definição da estrutura da personalidade avaliada pelo 16PF-5 é mais provável quando as parcelas dos itens são consideradas em uma análise fatorial, ao invés de diretamente o conjunto total de itens; isto seria esperado de acordo com os comentários de Cattell e Krug (1986). Porém, considerando o tamanho da amostra (mais de 3.000 participantes), o componente erro de medida que estes autores indicam como uma explicação da diferença dos resultados em função dos dois tratamentos da informação, deveria ser ponderado. A natureza complexa da estrutura da personalidade, discutida em parágrafos prévios, também ajuda a explicar a adequação de considerar as parcelas dos itens, ou mesmo os grupos de itens separadamente (ver Rolland \& Mogenet, 1996).

Finalmente, o leitor deve reconhecer a natureza e os critérios que orientaram o presente estudo. O propósito não foi comprovar a estrutura do 16PF-5, mas sim averiguar a possibilidade de se chegar a uma solução fatorial teoricamente esperada para este questionário (Cattell, 1946; 1973). Embora seja sugerida sua adequação ao contexto espanhol, paradoxalmente evidencia que unicamente 13 dos 16 fatores que preconiza atendem aos critérios estatísticos convencionados. O que dizer no caso da população brasileira? Segundo busca efetuada no Index Psi (2000), nenhuma publicação foi realizada sobre os parâmetros psicométricos desta medida; tudo o que se sabe tem sido disponibilizado pela instituição que a comercializa. Seria importante que pesquisadores independentes se adentrassem nesta temática, providenciando provas ou contraprovas a respeito.

\section{Agradecimentos}

Durante a preparação deste artigo o primeiro autor contou com uma bolsa de Produtividade em Pesquisa, do CNPq (Proc. 301705/91-7). Os autores agradecem a esta instituição e também a dois revisores anônimos, cujas contribuições foram fundamentais para melhorar a presente versão.

\section{Referências}

Adcock, N. V., \& Adcock, C. J. (1977). The validity of the 16 PF personality structure: a large New Zealand sample item analysis. Journal of Behavioral Science, 2, 227-237.

Adcock, N. V., Adcock, C. J., \& Walkey, F. H. (1974). Basic dimensions of personality. International Review of Applied Psychology, 23, 131-137.

Bolton, B. (1977). Evidence for the 16 PF primary and secondary factors. Multivariate Experimental Clinical Research, 3, 1-15.

Burdsal, C. A., \& Bolton, B. (1979). An item factoring of 16 PF-E: further evidence concerning Cattell's normal personality sphere. Journal of General Psychology, 100, 103-109.

Cattell, H. E. P. (1996). The original Big Five: a historical perspective. European Review of Applied Psychology, 46, 5-14. 
Cattell, R. B. (1946). The description and measurement of personality. Nova York: World.

Cattell, R. B. (1973). Personality and mood by questionnaire. São Francisco, CA: Jossey-Bass.

Cattell, R. B. (1975). Cuestionario de personalidad 16 PF. Madri: TEA Ediciones.

Cattell, R. B., \& Cattell, H. E. P. (1995). Personality structure and the new fifth edition of the 16PF. Educational and Psychological Measurement, 55, 926-937.

Cattell, R. B., \& Krug, S. E. (1986). The number of factor in the 16PF: a review of the evidence with special emphasis on methodological problems. Educational and Psychological Measurement, 46, 509-522.

Conn, S. R., \& Rieke, M. L. (1994). The 16PF fifth edition. Technical manual. Champaign, Illinois: Institute for Personality and Ability Testing.

Eysenck, H. J. (1972). Primaries or second order factors: a critical consideration of Cattell's 16 PF battery. British Journal of Social and Clinical Psychology, $11,265-269$.

Fabricatore, J., Azen, S. P., Schoentgen, S., \& Snibbe, H. (1978). Predicting performance of police officers using the Sixteen Personality Factors Questionnaire. American Journal of Community Psychology, 6, 63-70.

Fox, S., Haboucha, S., \& Yossi, D. (1981). The predictive validity of the Sixteen Personality Factors Questionnaire relative to three independent criterion measures of military performance. Educational and Psychological Measurement, 41, 515-521.

Gorsuch, R. L. (1983). Factor analysis. Hillsdale, New Jersey: Lawrence Erlbaum. Guilford, J. P. (1975). Factors and factors of personality. Psychological Bulletin, $82,802-814$.

Howarth, E., \& Browne, J. A. (1971). An item-factor analysis of the $16 \mathrm{PF}$ Personality, 2, 117-139.
Howarth, E., Browne, J. A., \& Marceau, R. (1972). An item analysis of Cattell's 16 PF. Canadian Journal of Behavioral Science, 4, 85-90.

Index Psi (2000). Base de dados para pesquisa. Endereço da página web: http://www.psicologia-online.org.br/index_psi.htm (Consultada em 13 de Agosto).

Jenrich, R. I., \& Sampson, P. F. (1966). Rotation for simple loadings. Psychometrika, 31, 313-323.

Lord, W. (1996). Use of the 16PF in UK: applications in the work place. European Review of Applied Psychology, 46, 67-72.

Pasquali, L. (1999). Testes referentes a construto: teoria e modelo de construção. In L. Pasquali (Org.), Instrumentos psicológicos: manual prático de elaboração (pp. 37-71). Brasília: LabPAM / IBAPP.

Prieto, J. M. (1980). Estructura de la personalidad humana a partir de datos $Q$ en adultos: estudio factorial con muestras españolas. Madri: Editorial Universidade Complutense.

Prieto, J. M., Gouveia, V. V., \& Fernández, M. A. (1996). Evidence on the primary source-trait structure in the Spanish 16PF, 5th edition. European Review of Applied Psychology, 46, 33-43.

Rolland, J.-P., \& Mogenet, J. L. (1996). Éléments de validité des dimensions primaires de l'adaptation française de l'inventaire 16PF5. European Review of Applied Psychology, 46, 25-30.

Russell, M. T., \& Karol, D. L. (1995). Manual 16 PF-5. Madri: TEA Ediciones.

Seisdedos, N. (1978). 16PF monografía técnica. Madri: TEA Ediciones.

Seisdedos, N. (1996). Introduction. European Review of Applied Psychology, $46,33-43$.

Tyler, B. (1996). Source and surface traits: structure and interpretation. European Journal of Applied Psychology, 46, 57-63.

1 Uma versão resumida deste estudo foi apresentada no VIII Simpósio Brasileiro de Pesquisa e Intercâmbio Científico, promovido pela ANPEPP, de 24 a 27 de Maio de 2000, Serra Negra, SP.

Valdiney V. Gouveia, doutor em Psicologia pela Universidade Complutense de Madri, é professor no departamento de Psicologia da Universidade Federal da Paraíba, bolsista pesquisador do CNPq e coordenador do grupo de pesquisa Bases Normativas do Comportamento Social. Endereço para correspondência: Universidade Federal da Paraíba, CCHLA-Departamento de Psicologia; João Pessoa, PB; CEP 58051-900. E-mail: vgouveia@cchla.ufpb.brou vvgouveia@uol.com.br José María Prieto, doutor em Psicologia pela Universidade Complutense de Madri, é professor no Departamento de Psicologia Diferencial da Universidade Complutense de Madri.. 